\title{
Benefit and risks of trivalent 2010 seasonal influenza vaccine in Australian children
}

R Lopert (ruth.lopert@tga.gov.au)1

1. Therapeutic Goods Administration, Australia

Citation style for this article:

Lopert R. Benefit and risks of trivalent 2010 seasonal influenza vaccine in Australian children.

Euro Surveill. 2010;15(40):pii=19679. Available online: http://www.eurosurveillance.org/ViewArticle.aspx?Articleld=19679

Article published on 7 October 2010

To the editor: In a recent issue of Eurosurveillance, Kelly et al. conclude that the risks associated with the 2010 trivalent influenza vaccine from CSL Biotherapies outweighed its benefits, based on a comparison of the risk of hospitalisation due to influenza compared with that arising from an adverse reaction [1]. However the analysis is misleading and the authors' conclusions must be considered unsound.

The authors fail to recognise the obvious and significant differences between being hospitalised for overnight observation following a febrile convulsion and being admitted to intensive care units in respiratory failure as a result of influenza infection. A study in the United States conducted in 2006 reported a $15 \%$ intensive care units admission rate in children in this age group hospitalised with influenza infection [2].

An Australian study demonstrated that for children hospitalised with influenza $12.3 \%$ had pneumonia, $7.4 \%$ required intensive care units admission for ventilatory support and $2.5 \%$ required inotropes [3]. In Australian children aged less than five years deaths from influenza have been reported at a rate of 0.2 per 100,000 children [4]. By contrast simple febrile seizures have not been shown to increase the risk of death, and although there is a small increase in risk associated with complex febrile seizures, this is very rare even in high-risk children $[5,6]$. A calculation of risks and benefits using the crude measure of rates of hospitalisation alone as applied by the authors fails to take into account the relevant mortality and morbidity rates associated with these admissions. In addition, it does not account for morbidity of influenza in the broader community.

Though not mentioned in the article, it might be relevant in any analysis of its conclusions to note that the authors were directly involved in the design and oversight of the population-based clinical trial that is the subject of the article (i.e. the administration of influenza vaccine to all children under the age of five years occurring only in Western Australia). The vast majority of adverse events referred to occurred in West Australian children participating in this trial in which influenza vaccination of young children was extended beyond the relevant national recommendations. The authors' observation that 'the benefit-risk profile would be improved if only children who were at increased risk of hospitalisation following influenza infection were targeted for vaccination' in fact describes the recommendations of the Australian Government's National Immunisation Program, that the use of seasonal vaccine in this age group be limited to those at increased risk of influenza infection. The baseline risk benefit calculation in this population is clearly quite different to the population involved in the Western Australian trial.

Because of the authors' methodological approach conclusions have been made about the risks and benefits of vaccines without consideration of all the relevant factors. However, while the lack of consideration of relevant factors must call into question the validity of the conclusions, the analysis may well give rise to reconsideration within Western Australia of the populationbased clinical trial.

Of even greater concern however is the unnecessary and damaging impact simplistic analyses can have on public confidence in childhood immunisation programmes in general and the consequences of immunisation recommendations being ignored out of fear and misinformation.

\section{References}

1. Kelly H, Carcione D, Dowse G, Effler P. Quantifying benefits and risks of vaccinating Australian children aged six month to four years with trivalent inactivated seasonal influenza vaccine in 2010. Euro Surveill. 2010;15(37):pii=19661. Available from: http://www.eurosurveillance.org/ViewArticle. aspx?Articleld=19661

2. Ampofo K, Gesteland PH, Bender J, Mills M, Daly J, Samore M, et al. Epidemiology, complications and costs of hospitalisation in children with laboratory-confirmed influenza infection. Pediatrics. 2006;118(6):2409-17.

3. Lester-Smith D, Zurynski YA, Booy R, Festa MS, Kesson AM, Elliott EJ. The Burden of childhood influenza in a tertiary paediatric setting. Commun Dis Intell. 2009;33(2):209-15.

4. Brotherton J, Wang H, Schaffer A, Quinn H, Menzies R, Hull B, et al. Vaccine preventable diseases and vaccination coverage in Australia, 2003 to 2005. Commun Dis Intell. 2007;31 Suppl:S1-152.

5. Chungath $M$, Shorvon $D$. The mortality and morbidity of febrile seizures. Nat Clin Pract Neurol. 2008;4(11):610-21.

6. Vestergaard M, Pedersen MG, Østergaard J, Pederson CB, Olsen J, Christensen J. Death in children with febrile seizures: a population based cohort study. Lancet. 2008;372(9637):457-63. 